wurden in der TG häufiger als stark belastend empfunden und Patienten fühlten sich dort auch selbst stärker unter Druck gesetzt.

\section{Indikation genau stellen}

Auch Professor Bernhard Strauß, Institut für Psychosoziale Medizin und Psychotherapie, Universitätsklinikum Jena, fand in seinen Untersuchungen, dass die Gruppe die Patienten noch stärker stresst als der Therapeut. Noch ist nicht klar, wie bedeutsam das für das Therapieer- gebnis ist, aber ein Ausscheiden aus der Gruppe wird häufig auf Faktoren in der Gruppe zurückgeführt. Belastungsunerschiede zwischen Gruppentherapien scheinen kaum relevant. Für Strauß ist es wichtig, wegen einer möglichen Überforderung durch die Gruppe die Indikation zur Gruppentherapie genau zu stellen und das Setting an die Möglichkeiten der Gruppenmitglieder anzupassen.

Auch Linden hält die Belastung durch die Gruppe für relevant: Demoralisierung und Therapieabwehr könnten den
Therapieerfolg nachhaltig beeinträchtigen. Die ungesteuerte Interaktion in der Selbsthilfegruppe werde nach seinen Untersuchungen als weniger belastend und nebenwirkungsträchtig erlebt. Dennoch ist für ihn klar: „Nebenwirkungen der ,guten' Therapie müssen wir aktuell in Kauf nehmen - solange wir nichts besseres haben."

Friederike Klein

Linden M. „Belastungserleben von Patienten in therapeutengeleiteten Gruppenpsychotherapien im Vergleich zu assoziierten Selbsthilfegruppen"; DGPPN-Kongress 2016, Berlin, 25.11.2016

\title{
Unbedingt jetzt schon vormerken! - 30. AGNP-Symposium in München im Herbst 2017
}

Wenn das 184. Oktoberfest in München zu Ende geht, beginnt am Tag darauf das 30. AGNP-Symposium an der Ludwig-Maximilians-Universität in München. Vom 4. bis 7. Oktober 2017 bekommen Fachkollegen, Ärzte und auch nicht ärztliche Berufsgruppen ein Programm geboten, das zahlreiche aktuell brisante Themen und Bedarfe rund um die Psychiatrie beleuchtet.

Die Psychiatrie steht im klinischen und im wissenschaftlichen Bereich vor großen Herausforderungen. Insbesondere muss unter den veränderten Bedingungen eines sich abzeichnenden neuen Vergütungssystems, dessen Form und Inhalt noch nicht abschließend feststehen, auch die klinische Forschung weiterhin sichergestellt werden. Gleichzeitig kündigen sich ebenfalls fundamentale Veränderungen in der Interaktion mit der pharmazeutischen Industrie ab, die einerseits immer weniger Forschungsvorhaben von pharmakologisch interessierten Psychiatern fördern kann, und sich andererseits gegenwärtig in einer Krise hinsichtlich der Entwicklung innovativer Präparate befindet. Zusätzlich wird vermehrt Kritik an dem bisherigen Einfluss von Wirtschaftsunternehmen auf die universitäre Forschung laut. Vor diesem Hintergrund wird das Symposium ganz ohne "Sponsoring" durch Pharmaunternehmen organisiert werden. Diese Änderung der "Sponsoring"-Kultur soll aber nicht in dem Sinne missverstanden werden, dass einer Zusammenarbeit zwischen forschenden Pharmaunternehmen und universitärer Psychiatrie kein hoher Stellenwert mehr eingeräumt wird. Gerade die nachgewiesene Unabhängigkeit psychiatrischer Forschung kann die erforderliche Zusammenarbeit mit pharma- zeutischen Unternehmen am besten unterstützen.

Eine erfreuliche Entwicklung ist die Tatsache, dass mittlerweile Entwicklungsstörungserkrankungen nun auch in der Erwachsenenpsychiatrie als Störungen von hoher klinischer Relevanz akzeptiert werden und gleichzeitig störungsübergreifende Konzepte wie Veränderungen der zirkadianen Rhythmik zunehmend in den Fokus wissenschaftlicher Bemühungen kommen. Das 30. AGNP-Symposium bietet ein Forum, die Herausforderungen und Chancen zu diskutieren, die sich durch diese neuen Perspektiven ergeben. Das 30. AGNP-Symposium bietet ebenso, wie gewohnt, die Möglichkeit, eigene Forschungsergebnisse aus Klinik und Grundlagenforschung vorzustellen und mit einem breiten Fachkollegium aus dem In- und Ausland zu diskutieren.

Zum Programm gehören erstmals auch Fortbildungskurse für verschiedene in Klinik und Forschung tätige nicht ärztliche Berufsgruppen. Wir wollen Mitarbeiterinnen und Mitarbeitern, die in vielen Forschungslabors, aber auch in der klinischen Forschung, beispielsweise als technische Assistenten und "study nurses" hervorragende Arbeit leisten, adäquate Weiterbildungsangebote bieten.
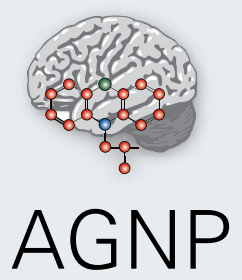

Arbeitsgemeinschaft für Neuropsychopharmakologie und Pharmakonsvchiatrie

Der Vorstand und das Programmkomitee der AGNP (Arbeitsgemeinschaft für Neuropsychopharmakologie und Pharmakopsychiatrie) laden herzlich ein zum 30. AGNP-Symposium in München.

Das Einreichen von Vorschlägen für Symposien/Workshops/Pro-Con-Debatten ist bereits ab sofort möglich (die entsprechenden Formulare sind auf der Homepage der AGNP erhältlich). Abstracts für Poster und freie Vorträge können ebenfalls bereits online vorgelegt werden.

Anmeldung und weitere Informationen: https://kongress.agnp.de/

Jens Wiltfang, 1. Vorsitzender der AGNP

Johannes Thome,

3. Vorsitzender der AGNP, Programmkomitee Golo Kronenberg, Programmkomitee

Und noch ein Terminhinweis!

AGNP-Psychopharmakologie-Tage unter der wissenschaftlichen Leitung von Prof. Dr. med. Borwin Bandelow

16. - 17. Juni 2017 in Berlin im Seminaris Campushotel Berlin, Takustr. 39

www.agnp.de / info@agnp.de 